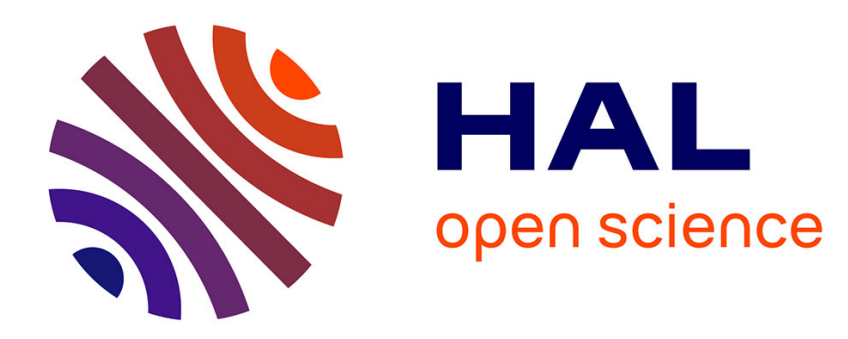

\title{
Adaptation des bovins aux milieux d'élevage
}

P. Le Neindre, J.P. Garel

\section{- To cite this version:}

P. Le Neindre, J.P. Garel. Adaptation des bovins aux milieux d'élevage. Productions Animales, 1990, 3 (3), pp.189-194. hal-00895901

\section{HAL Id: hal-00895901 https://hal.science/hal-00895901}

Submitted on 1 Jan 1990

HAL is a multi-disciplinary open access archive for the deposit and dissemination of scientific research documents, whether they are published or not. The documents may come from teaching and research institutions in France or abroad, or from public or private research centers.
L'archive ouverte pluridisciplinaire HAL, est destinée au dépôt et à la diffusion de documents scientifiques de niveau recherche, publiés ou non, émanant des établissements d'enseignement et de recherche français ou étrangers, des laboratoires publics ou privés. 
INRA Prod. Anim., 1990, 3 (3), $189-194$

\section{P. LE NEINDRE, J.P. GAREL *}

INRA Laboratoire de I'Elevage Bovin Theix 63122 Saint-Genès-Champanelle

* INRA Domaine de la Borie

\section{Marcenat}

15190 Condat

\section{Adaptation des bovins aux milieux d'élevage}

Les veaux Salers sont habituellement allaités par leurs mères alors que les veaux Pie-Noirs en sont séparés dès la naissance. Pour chaque race, les animaux sont " adaptés » à ces conditions d'élevage. Quelles sont les parts respectives de la race et du mode d'élevage dans le jeune âge sur cette adaptation?

Les animaux des races laitières, à viande ou rustiques sont élevés dans des milieux très contrastés. Il est généralement admis que ces animaux ont des capacités physiologiques et comportementales différentes qui leur permettent, en plus de leurs capacités à produire du lait ou de la viande, d'être adaptés à leurs milieux d'élevage habituels.

Par exemple, les vaches traites doivent produire le maximum de lait à la traite et donc s'adapter à la machine à traire. En revanche, les vaches à viande ne doivent allaiter que leurs veaux.

Les jeunes de l'élevage laitier sont séparés de leur mère dès la naissance. Ils doivent boire le lait au seau et ingérer rapidement des aliments solides. Ceux des races à viande sont allaités pendant une période très longue et sont donc beaucoup moins dépendants de l'homme.

Cette notion d' « adaptation » est assez fréquemment évoquée en particulier par les généticiens travaillant sur les ruminants (Bibé et Vissac 1979, Bougler et al 1988 ). Ces chercheurs s'interrogent sur les bases génétiques et physiologiques de l'adaptation des animaux à

\section{Résumé}

Des veaux de races Pie-Noire et Salers ont été observés dans différentes conditions d'élevage et à différentes périodes de leur vie. S’ils sont avec leur mère dès la naissance, leurs activités ne sont pas statistiquement différentes entre les deux races. En revanche lorsqu'ils sont seuls, les veaux Pie-Noirs sont plus actifs que les Salers, ils boivent plus facilement le lait au seau, ils ingèrent plus d'aliment solide et ont finalement une meilleure croissance. La précocité sexuelle des PieNoires est supérieure à celle des Salers. Ces différences peuvent être interprétées en termes d'adaptations aux différents milieux d'élevage. Le terme " rusticité ». trop imprécis, devrait être évité. leur milieu et sur les interactions entre le génotype et le milieu. La définition de l'adaptation est essentielle, en particulier, pour comprendre ce que sous entend la rusticité. Ce terme est parfois utilisé pour définir la capacité des animaux à s'adapter à une grande variété de situations. Parfois il ne signifie que la capacité des animaux à survivre et à se reproduire dans des conditions climatiques difficiles.

Trois séries d'expérimentations nous ont permis de comparer des animaux de deux races supposées très différentes: la race Pie-Noire, laitière, et la race Salers, "rustique », dans les conditions habituelles d'élevage des jeunes pour chacune de ces races et donc d'analyser l'interaction entre la race et le mode d'élevage.

\section{1 / Vitalité des veaux après la naissance}

La première série d'observations porte sur l'activité des veaux dans les heures qui suivent la naissance. Leur vilalité est caractérisée par l'intervalle de temps entre la naissance et la première station debout.

Cinquante-six veaux, de races Pie-Noire $(\mathrm{n}=$ $25)$ et Salers $(n=31)$, ont été observés pendant les 4 premières heures suivant leur naissance. Ils étaient soit laissés avec leur mère comme cela se pratique dans les élevages allaitants (maternés) soit isolés dès la naissance comme dans les élevages laitiers (isolés).

Les veaux avec leur mère se sont levés beaucoup plus rapidement que ceux qui étaient isolés (figure 1), qu'ils soient de race Pie-Noire ou Salers. En revanche, lorsqu'ils étaient isolés, une grande partie des veaux Salers $(60 \%)$ ne se sont pas levés pendant les 4 heures d'observa- 
Figure 1. Influence de la race des veaux et de leur environnement après la naissance sur l'intervalle de temps entre la naissance et la première station debout. L'intervalle est pris égal à 4 heures si le veau ne s'est pas levé pendant la période d'observation de 4 heures.

Intervalle moyen $(h) \pm$ écart-type

(nombre de veaux qui se sont levés/nombre total)

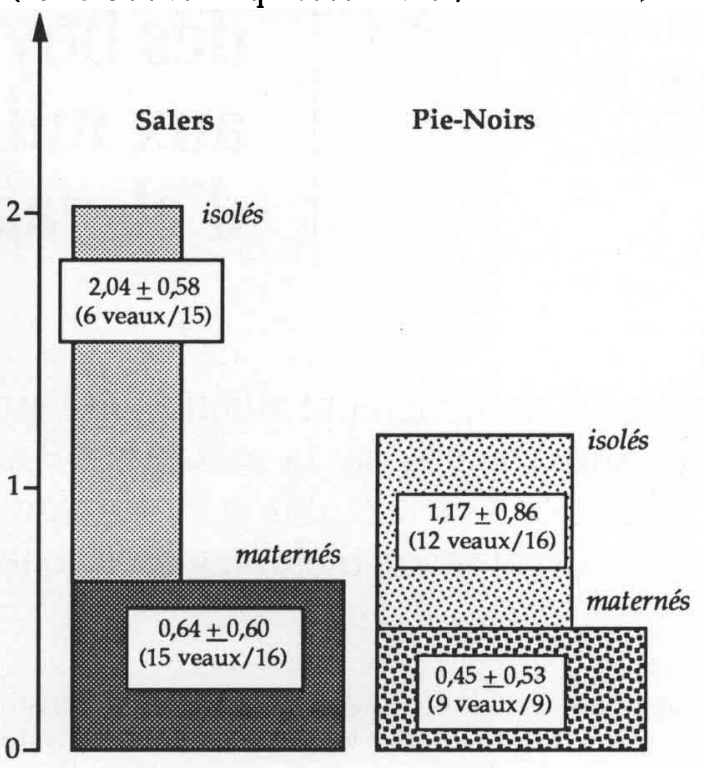

tion. Le temps pris pour se lever observé chez les Salers a été beaucoup plus important que celui obtenu chez les Pie-Noirs (respectivement $2,04 \mathrm{~h}$ et $1,2 \mathrm{~h}$ ). Donc la privation de la mère affecte de façon beaucoup plus marquée la mobilité des nouveau-nés chez les Salers que chez les Pie-Noirs.

Huit veaux Salers maternés et 8 veaux PieNoirs isolés ont reçu, au biberon, des quantités non limitées de colostrum 4 heures et 10 heures après leur naissance. Des prélèvements sanguins effectués régulièrement ont permis de déterminer le taux sanguin maximum d'immunoglobulines. Les veaux Pie-Noirs isolés ont consommé, lors des deux repas, des quantités de colostrum plus importantes que les Salers maternés (+ $80 \%$ ). Leur taux maximum d'immunoglobulines a été également beaucoup plus élevé que celui des Salers (+ $45 \%$ ) (tableau 1). Par conséquent, même dans des conditions plus défavorables, c'est-à-dire en l'absence de la mère, les capacités de consommation et d'absorption des immunoglobulines du colostrum sont plus élevées chez les Pie-Noirs.

\section{2 / Aptitude des veaux à boire au seau}

Dix-neuf veaux Pie-Noirs et 13 Salers ont été séparés de leur mère dès la naissance et ont été nourris artificiellement. Chaque veau recevait $\mathrm{du}$ colostrum au biberon lors des deux premiers repas. Par la suite, à chacun des deux repas journaliers, du lait était proposé au veau au biberon pendant 30 secondes puis dans un seau pendant 10 minutes. L'animal ne bénéficiait d'aucune aide pour l'amener à boire au seau. Les veaux qui n'avaient pas bu au seau pendant la période de 10 minutes étaient alimentés au biberon. On a considéré qu'un veau savait boire au seau lorsqu'il buvait dans le seau lors de deux repas consécutifs.

La moitié des veaux Pie-Noirs ont bu dès le premier repas avec un seau et $88 \%$ buvaient lors du sixième repas. En revanche, aucun veau Salers n'a bu avant le quatrième repas; seulement $30 \%$ des veaux avaient bu lors du 12 repas (figure 2). Très clairement, les nouveaunés Pie-Noirs présentent une capacité d'adaptation à la buvée au seau bien supérieure à celle des Salers.

\section{3 / Capacité d'ingestion et croissance des jeunes veaux}

Des veaux femelles de races Salers $(\mathbf{n}=25)$ et Pie-Noire $(n=29\}$, nés en moyenne au mois de janvier, ont été soit isolés dès la naissance et nourris artificiellement soit maternés par une nourrice.

Dans le cas de l'allaitement artificiel, $40 \mathrm{~kg}$ de poudre de lait ont été distribués sur une période d'environ 57 jours après la naissance. Le lait était distribué deux fois par jour dans un seau muni d'une tétine afin de ne pas défavoriser les veaux de race Salers (voir paragraphe 2).

Tableau 1. Quantités de colostrum ingérées au biberon et taux sanguin d'immunoglobulines de veaux Salers maternés et de veaux Pie-Noirs isolés. (Levieux et al, résultats non publiés).

\begin{tabular}{|c|c|c|c|}
\hline & $\begin{array}{c}\text { Salers } \\
\text { maternés }\end{array}$ & & $\begin{array}{l}\text { Pie-Noirs } \\
\text { isolés }\end{array}$ \\
\hline Nombre de veaux & 8 & & 8 \\
\hline $\left.\begin{array}{l}\text { Quantités de colostrum ingéré } \\
-4 \text { heures } \\
-10 \text { heures }\end{array}\right\} \begin{array}{l}\text { après la } \\
\text { naissance }\end{array}$ & $\begin{array}{l}1,7 \\
0,8\end{array}$ & $\begin{array}{l}\text { * } \\
\text { * }\end{array}$ & $\begin{array}{l}2,9 \\
1,6\end{array}$ \\
\hline $\begin{array}{l}\text { Quantités d'immunoglobuline } \\
\text { ingérées (g) }\end{array}$ & 186 & * & 331 \\
\hline $\begin{array}{l}\text { Taux sanguin maximum } \\
\text { d'immunoglobuline }(\mathrm{mg} / \mathrm{kg})\end{array}$ & 28,0 & * & 40,7 \\
\hline
\end{tabular}

* différence significative à $\mathrm{P}<0,05$. 
Figure 2. Adaptation à la buvée au seau de veaux Salers ou Pie-Noirs.

veaux qui boivent au seau (\%)

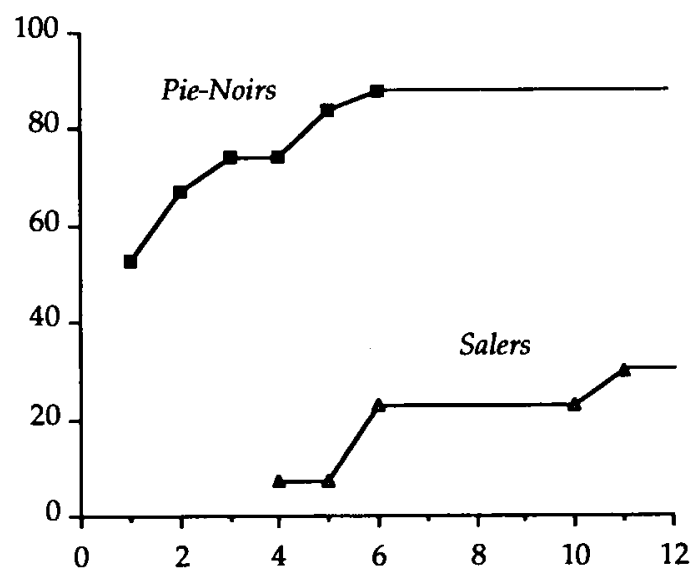

nombre de distributions au seau depuis la naissance

En plus du lait, les veaux recevaient à volonté du foin et de l'aliment concentré. Ils sont restés isolés jusqu'à la troisième semaine après le sevrage. Ils ont ensuite été regroupés dans des parcs en stabulation libre et ont reçu du foin à volonté et $3 \mathrm{~kg}$ d'aliment concentré par jour. $\mathrm{Au}$ printemps, ces veaux ont été mis à l'herbe où ils recevaient $2 \mathrm{~kg}$ d'aliment concentré par jour.

Les veaux allaités étaient adoptés le plus rapidement possible après la naissance par une vache de race Salers. Ils ont tété sous surveillance deux fois par jour pendant la période hivernale. Pendant la période estivale et jusnourrice au pâturage. A l'automne, tous ces animaux ont été mis en stabulation libre où ils ont reçu du foin et un aliment concentré. A la qu'au sevrage à l'automne ils étaient avec leur

fin du second hiver ils sont allés de nouveau au pâturage.

Les quantités de foin et d'aliment concentré ingérées par les veaux élevés sans la mère ont été mesurées de la naissance jusqu'au $21^{\mathrm{e}}$ jour après le sevrage.

Les quantités de lait ingérées par les veaux élevés sous des nourrices ont été obtenues toutes les deux semaines par pesées des jeunes avant et après la tétée (Le Neindre 1973).

Lorsqu'ils étaient allaités, les veaux des deux races ont eu la même croissance. En revanche, dans le cas de l'allaitement artificiel la croissance des veaux Pie-Noirs a été significativement supérieure de $100 \mathrm{~g}$ par jour environ à celle des Salers. Cette différence est due probablement aux faibles quantités de foin et d'aliment concentré ingérées par ces derniers (tableau 2). A 9 mois, les génisses Salers élevées sous une nourrice pesaient $54,5 \mathrm{~kg}$ de plus que leurs homologues élevées sans nourrice alors que la différence observée pour la race Pie-Noire n'était que de $18,6 \mathrm{~kg}$. A l'âge d'environ 21 mois, juste avant la mise à la reproduction, les écarts étaient encore respectivement de 49,4 et $28,0 \mathrm{~kg}$ (figure 3 ). Donc, en conditions d'élevage sans la mère, les performances des Pie-Noires, tant du point de vue des capacités d'ingestion que de la croissance, sont meilleures que celles des Salers, alors qu'il n'y a pas de différence en allaitement maternel (au moins en ce qui concerne la croissance).

\section{4 / Précocité sexuelle}

L'existence de cycles oestriens a été déterminée à 6 reprises entre les âges de 4 et 18 mois. A chaque fois, le taux de progestérone a été mesuré sur deux échantillons de sang prélevés à dix jours d'intervalle. Une quantité impor-

Tableau 2. Consommation et croissance de veaux femelles sevrés précocement ou élevés au pis.

\begin{tabular}{|c|c|c|c|c|}
\hline \multirow[b]{2}{*}{ Race } & \multicolumn{2}{|c|}{$\begin{array}{l}\text { Sevrage précoce } \\
\text { sans la nourrice }\end{array}$} & \multicolumn{2}{|c|}{$\begin{array}{l}\text { Sevrage tardif } \\
\text { avec une nourrice }\end{array}$} \\
\hline & Pie Noire & Salers & Pie Noire & Salers \\
\hline Nombre de veaux & 14 & 12 & 15 & 13 \\
\hline Quantité de lait bu $(\mathrm{kg})$ & $40(a)$ & $40(a)$ & $\begin{array}{c}1670 \pm 226 \\
(6,3 / \text { jour })\end{array}$ & $\begin{array}{c}1733 \pm 219 \\
(6,4 / \text { jour })\end{array}$ \\
\hline Age au sevrage (j) & 56,3 & 57,0 & $265 \pm 26$ & $271 \pm 17$ \\
\hline $\begin{array}{l}\text { Quantités d'aliments solides ingérées ( } \mathrm{kg} \text { ) } \\
\text { avant le sevrage des animaux isolés } \\
\text { - de foin } \\
\text { - d'aliment concentré } \\
\text { Quantités d'aliments solides ingérées ( } \mathrm{kg} \text { ) } \\
\text { après le sevrage des animaux isolés ( } 21 \text { jours) } \\
\text { - de foin } \\
\text { - d'aliment concentré }\end{array}$ & $\begin{array}{r}4,6 \pm 3,3 \\
14,3 \pm 10 \\
\\
6,2 \pm 4,8 \\
43,9 \pm 9,1\end{array}$ & $\begin{array}{l}6 \pm 1,4 \\
8,5 \pm 5,9 \\
, 5 \pm 1,8 \\
1,1 \pm 2,8\end{array}$ & & \\
\hline $\begin{array}{l}\text { Gain de poids vif entre la naissance } \\
\text { et } 3 \text { mois }(\mathrm{g} / \mathrm{j})\end{array}$ & $758 \pm 110$ & $56 \pm 102$ & $717 \pm 132$ & $768 \pm 114$ \\
\hline
\end{tabular}

(a) quantité de poudre de lait

$\star \star \star$ écart significatif à $P<0,001$

\section{En allaitement artificiel, la croissance des veaux Pie-Noirs est supérieure à celle des Salers, alors qu'elle est la même en allaitement maternel.}


Figure 3. Influence de la race et du mode d'élevage dans le jeune âge sur le poids, de la nais-

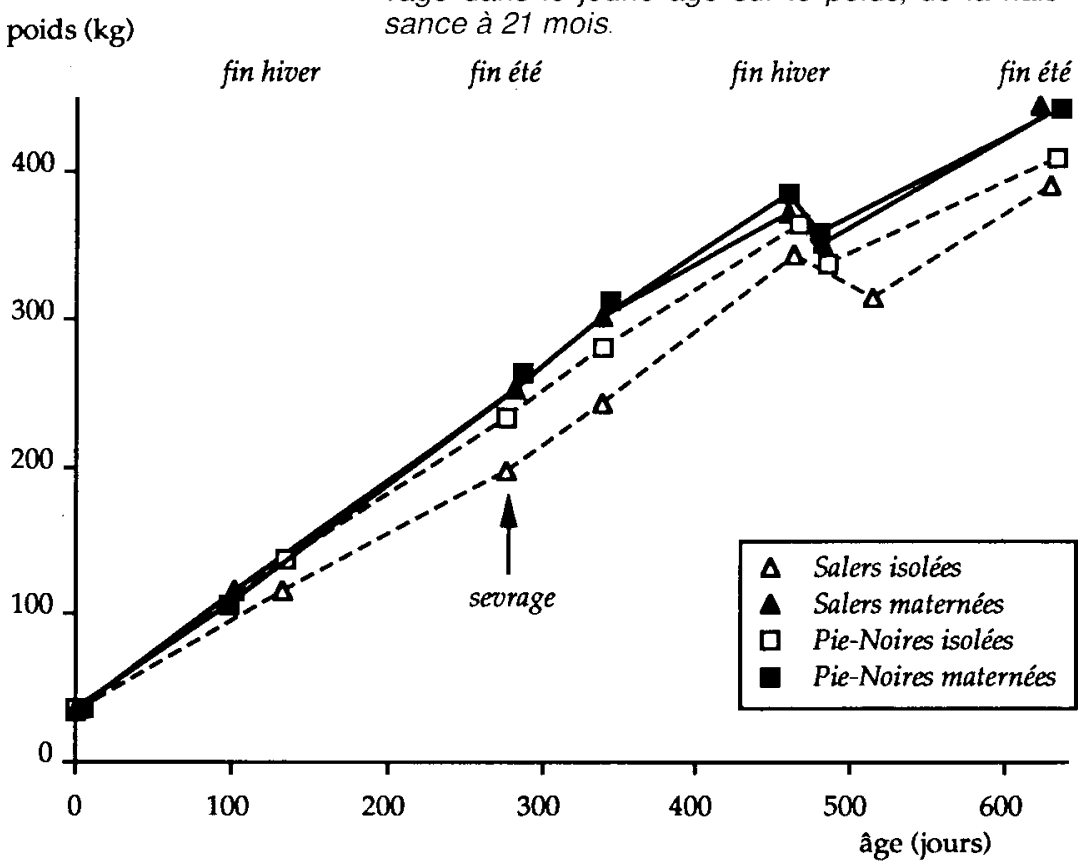

Figure 4. Influence de la race et du mode d'élevage dans le jeune âge sur la cyclicité de génisses entre 4 et 18 mois.

génisses cyclées $(\%)$

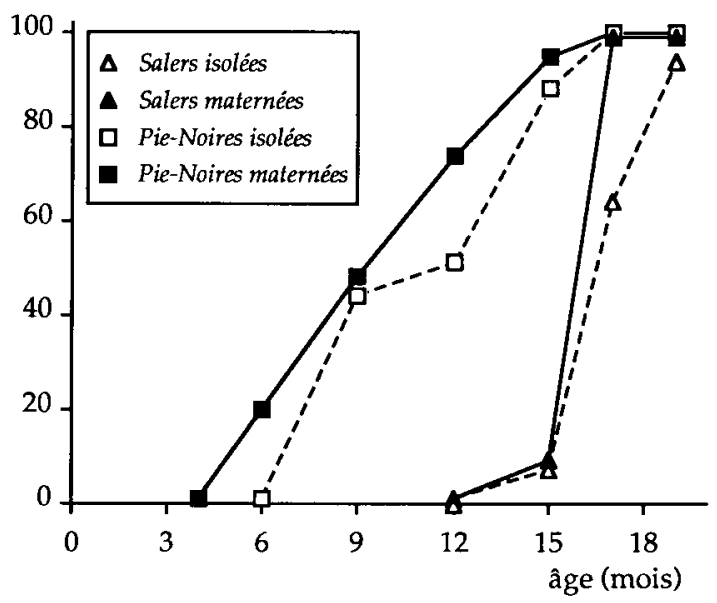

tante de progestérone dans un au moins des deux échantillons permet de diagnostiquer un cycle. Deux génisses Pie-Noires étaient gestantes au sevrage à 9 mois, nous avons donc considéré qu'elles étaient cycliques après cette date pour comparer les différents lots.

Dès l'âge de 6 mois quelques génisses PieNoires étaient cycliques et à un an plus de la moitié d'entre elles l'étaient. Aucune génisse Salers n'a été cyclique avant l'âge de 15 mois (figure 4).

\section{Discussion}

Des différences importantes entre races sont donc apparues pendant la période d'élevage.
Elles portent sur :

- la précocité : les génisses Pie-Noires ont été cycliques plus précocement que les Salers. Le développement précoce des animaux de race Pie-Noire a déjà été décrit dans le cadre d'études sur le développement corporel (Geay et Malterre 1973). La précocité sexuelle des PieNoires est connue depuis longtemps. Notre étude fait apparaître clairement que cette différence n'est pas liée au mode d'élevage.

- la capacité d'ingestion : les animaux Pie-Noirs ont ingéré plus d'aliments solides que les Salers pendant leurs trois premiers mois de vie. Cette différence avait déjà été décrite chez des génisses plus âgées de ces deux races (Agabriel et al 1987). Les écarts de poids liés au mode d'élevage sont restés beaucoup plus importants pour les Salers que pour les Pie-Noires jusqu'à l'âge de 20 mois.

D’autres différences ont été observées qui ne sont pas forcement imputables à la précocité ou à la capacité d'ingestion des animaux. Ainsi les Pie-Noirs sont plus actifs que les Salers à la naissance. L'activité d'un certain nombre de nouveau-nés d'espèces sauvages est très limitée en l'absence de la mère. Cette aptitude à se cacher (hider) et à rester immobile (freezing) permettrait de réduire la prédation (Lent 1974). La nécessité d'avoir des animaux actifs quand ils sont seuls, pour pouvoir être élevés artificiellement, a peut-être amené à éliminer, ou au moins à réduire, la tendance au freezing chez le jeune Pie-Noir. La faible activité des veaux Salers ne serait donc pas forcément la conséquence de l'état de maturation physiologique ou comportemental impliqué dans la notion de précocité. D'autre part la buvée est caractérisée par le pompage du liquide alors que la buvée suppose une succion ; ce sont donc des mécanismes très différents et complexes. Les différences observées entre les veaux des deux génotypes peuvent être liées à l'exploration du milieu comme à la précocité ou à une anatomie particulière.

Ces quelques résultats montrent que les races laitières et à viande ne diffèrent pas uniquement sur leurs capacités à produire du lait ou de la viande mais sur un ensemble de caractéristiques de la vie de l'animal. Les exemples présentés ici concernaient plus spécifiquement les jeunes femelles. D'autres textes rapportent des différences entre ces deux races dans le comportement social et la réactivité (Le Neindre 1989a) et dans les relations mère-jeune (Le Neindre 1989b).

Il serait intéressant de savoir si ces différences ont été le fruit de la sélection consciente ou inconsciente de la part des éleveurs ou si elles sont le fruit du hasard. S'il s'agit d'une sélection due aux éleveurs, celà implique qu'il est possible de sélectionner de façon efficace sur les traits des caractères ci-dessus. Dans le cas contraire, ces résultats soulignent pour le moins que la sélection sur des paramètres de type production de viande ou de lait est susceptible d'avoir des conséquences indirectes sur d'autres aspects, y compris comportementaux, dont il serait utile de tenir compte dans l'avenir. La valeur adaptative des caractéristiques ainsi modifiées est également difficile à apprécier. Celle de la précocité sexuelle, par exem- 


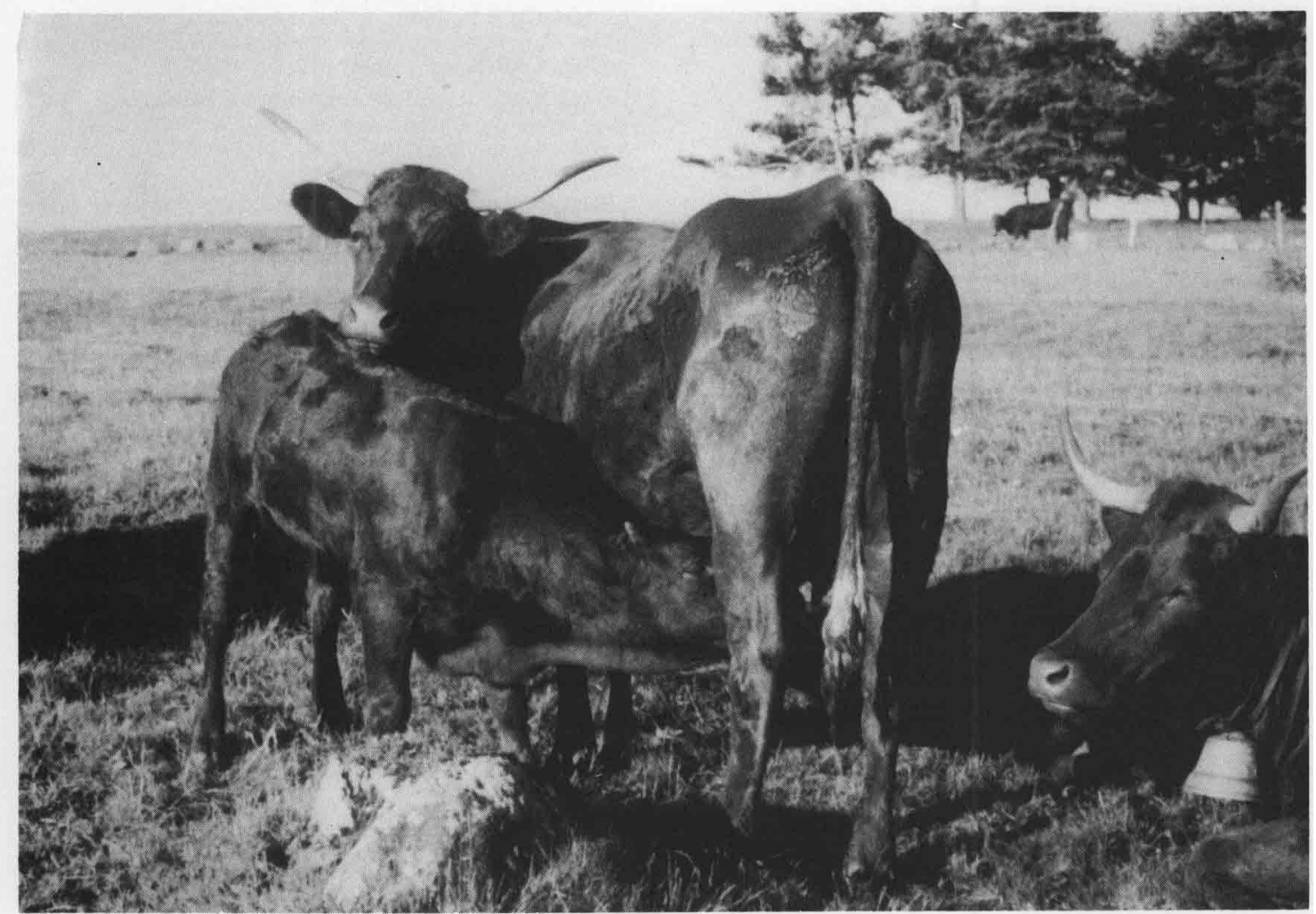

Les Salers, animaux de race rustique, sont adaptés à des conditions naturelles rigoureuses (climat,...) mais leurs performances diminuent lorsque les contraintes sont imposées par l'homme, comme par exemple l'élevage isolé de la mère.

ple, semble varier avec le mode d'élevage. Les éleveurs des troupeaux laitiers recherchent des animaux sexuellement précoces. En revanche, dans les conditions des élevages français de vaches allaitantes, l'apparition de la cyclicité chez les jeunes femelles avant leur sevrage entraîne des gestations précoces. Celles-ci conduisent bien souvent à l'élimination de ces animaux du fait de leur faible poids et de leurs problèmes ultérieurs de fertilité.

Les critères retenus dans ce texte, vitalité à la naissance et capacité d'ingérer des aliments solides, sont souvent présentés comme des critères de rusticité. Or, les Salers, qui sont des animaux de race rustique, ont eu de plus faibles performances que les animaux laitiers souvent présentés comme peu rustiques du fait de leur sélection dans les élevages " intensifs ».

En fait, l'adaptation à un milieu difficile, qui peut être une des définitions de la rusticité, ne signifie pas forcément une grande activité à la naissance ou une forte capacité d'ingestion mais peut-être une bonne résistance aux agressions climatiques et une bonne utilisation des ressources fourragères. Dans les conditions normales de l'élevage pour les nouveau-nés de race Salers, c'est-à-dire avec leur mère, ils se lèvent assez rapidement pour ingérer le colostrum et donc pour absorber les immunoglobulines nécessaires à leur survie.

Le milieu peut donc être difficile du fait des contraintes naturelles mais également de celles imposées par l'homme. L'élevage laitier est effectivement un milieu s'éloignant des conditions naturelles et, en particulier, le veau doit vivre sans sa mère. Dans ces conditions, le jeune Pie-Noir est vigoureux à la naissance et boit au seau. Cependant, cela ne signifie pas pour autant qu'il se comporte médiocrement lorsqu'il est avec sa mère. Son éventail comportemental est donc plus important. Il pourrait s'adapter dans plus de milieux, il serait plus flexible.

Plutôt que de rusticité, il serait donc préférable de parler d'adaptations à des milieux d'élevage différents. Ces adaptations portent sur de nombreuses caractéristiques aussi bien physiologiques que comportementales. Alors qu'elles semblent limitées strictement à un milieu spécifique dans le cas des espèces sauvages, la domestication semble avoir introduit une plus grande adaptabilité aux milieux imposés par l'homme. Cependant, on ne sait pas dans quelle mesure ces capacités nouvelles ont limité celles qui étaient caractéristiques des espèces sauvages. A l'intérieur d'espèces domestiques comme l'espèce bovine, des différences semblent exister entre populations, ou races, sur ces différents critères.

\section{Références bibliographiques}

AGABRIEL J., D'HOUR P., PETIT M., 1987. Influence de l'âge et de la race sur la capacité d'ingestion des femelles bovines. Reprod. Nutr. Dévelop., 27, 211-212.

BIBE B., VISSAC B., 1979. Amélioration génétique et utilisation du territoire. In G. Molénat et R. Jarrige Ed.: Utilisation par les ruminants des pâturages d'altitude et parcours méditerranéens. INRA-CRZV, Beaumont, 481-491.

BOUGLER J. BIBE B, VERRIER E, 1988. Choix et sélection d'un matériel génétique animal adapté ou adaptable aux systèmes extensifs. AFZ, 16 rue Claude-Bernard 75231 Paris, Cedex 05 , session d'hiver 
GEAY Y., MALTERRE C., 1973. Croissance, rendement et compsition des carcasses de jeunes bovins de différentes races. Bull. Tech. CRZV Theix, INRA, 14, 17-20.

LE NEINDRE P., 1973. Observations sur l'estimation de la production laitière des vaches allaitantes раг la pesée du veau avant et après la tétée. Ann. Zootech., 22, 413-422.

LE NEINDRE P., 1984. La relation mère-jeune chez les bovins : Influence de l'environnement social et de la race. Thèse d'état, Univ. Rennes, $274 \mathrm{pp}$.
LE NEINDRE P., 1989a. Influence of cattle rearing conditions and breed on social relationships of mother and young. Appl. Anim. Behav. Sci., 23, 117-127.

LE NEINDRE P., 1989b. Influence of rearing conditions and breed on social behaviour and activity of cattle in novel environments. Appl. Anim. Behav, Sci., 23, 129140.

LENT P.C., 1974. Mother-infant relationships in ungulates. in : V. Geist and F. Walther Eds: The behaviour of ungulates and its relation to management. Morgen, Switzerland, new series $24,14-55$.

\section{Summary}

Cattle adaptations to different husbandry systems.

Calves from a dairy breed (Friesian) and a French « hardy" breed (Salers) were compared in different rearing conditions (including artificial feeding vs. suckling) and at various ages. Animals from the two breeds were not different when reared by cows. Significant differences occurred when they were in a artificial rearing system. Friesian calves were more active than Salers at birth, drank milk more easily from a bucket, ate more feed and had a higher growth rate. Puberty occurred earlier in Friesians than in Salers. These results may be the consequence of adaptations differences to different husbandry systems. " Hardiness " seems to be a too general word to usefully describe these characteristics.

LE NEINDRE P., GAREL I.P., 1990. Adaptation des bovins aux milieux d'élevage. INRA Prod. Anim., 3 (3), $189-194$ 